Journal of Data Science 105-128, DOI: 10.6339/JDS.201801_16(1).0007

\title{
POWER LOMAX POISSON DISTRIBUTION: PROPERTIES AND ESTIMATION
}

\author{
Amal S. Hassan ${ }_{2}^{1}$ Said G. Nassr ${ }^{2}$ \\ ${ }^{I}$ Department of Mathematical Statistics, Cairo University \\ ${ }^{2}$ Faculty of Business Administration and International Marketing, Sinai University
}

\begin{abstract}
A new four-parameter lifetime distribution named as the power Lomax Poisson is introduced and studied. The subject distribution is obtained by combining the power Lomax and Poisson distributions. Structural properties of the power Lomax Poisson model are implemented. Estimation of the model parameters are performed using the maximum likelihood, least squares and weighted least squares techniques. An intensive simulation study is performed for evaluating the performance of different estimators based on their relative biases, standard errors and mean square errors. Eventually, the superiority of the new compounding distribution over some existing distribution is illustrated by means of two real data sets. The results showed the fact that, the suggested model can produce better fits than some well-known distributions.
\end{abstract}

Key words: Power Lomax distribution, Poisson distribution, Maximum likelihood, Moments, Probability Weighted moments, Least squares, and Weighted Least squares.

\section{Introduction}

Lomax (1954) suggested an important model for lifetime analysis called Lomax (Pareto type II) distribution. Its widely applied in some areas, such as, analysis of income and wealth data, modeling business failure data, biological sciences, model firm size and queuing problems, reliability modeling and life testing (see Harris (1968), Atkinson and Harrison (1978), Holland et al. (2006), Corbellini et al. (2007), Hassan and Al-Ghamdi (2009) and Hassan et al. (2016), respectively.

A random variable has the Lomax distribution with shape parameter and scale parameter if it has the probability density function (pdf) given by

The cumulative distribution function (cdf) corresponding to (1) is as follows 
Generalizations of the Lomax distribution have been formulated by several authors. For example, Ghitany et al. (2007) proposed a new distribution using the Marshall-Olkin generator. Abdul-Moniem and Abdel-Hameed (2012) introduced the exponentiated Lomax by adding a new shape parameter to the Lomax distribution. Lemonte and Cordeiro (2013) investigated beta Lomax, Kumaraswamy Lomax and McDonald Lomax. Cordeiro et al. (2013) introduced the gamma-Lomax using the gamma generator. The Weibull Lomax using Weibull generator has been proposed by Tahir et al. (2015). Also, the Gumbel-Lomax using Gumbel-X generator has been introduced by Tahir et al. (2016).

Rady et al. (2016) proposed a recent development called the power Lomax (PL) distribution as a new extension of the Lomax distribution by considering the power transformation

$Y=T^{\frac{1}{\beta}}$, where the random variable $T$ follows Lomax distribution with parameters $\alpha$ and $\lambda$. The pdf of the power Lomax distribution is defined by

$$
g(y ; \alpha, \lambda, \beta)=\alpha \beta \lambda^{\alpha} y^{\beta-1}\left(\lambda+y^{\beta}\right)^{-\alpha-1} ; \quad y, \alpha, \beta, \lambda>0 .
$$

The cdf of the power Lomax distribution is as follows

$$
G(y ; \alpha, \lambda, \beta)=1-\lambda^{\alpha}\left(\lambda+y^{\beta}\right)^{-\alpha} .
$$

In the literature, several authors proposed a new distribution to model lifetime data by combining some discrete distribution together with other known continuous distributions. Adamidis and Loukas (1998) proposed the two-parameter exponential-geometric distribution with decreasing failure rate. Following the same idea, Kus (2007) introduced the exponentialPoisson distribution with decreasing failure rate and discussed several of its properties. Chahkandi and Ganjali (2009) proposed the exponential power series family of distributions with decreasing failure rate which contains as special cases the exponential Poisson, exponential geometric and exponential logarithmic distributions. A three-parameter Weibull power series distribution with decreasing, increasing, upside-down bathtub failure rate functions has been introduced by Morais and Barreto-Souza (2011). Based on the exponential geometric distribution, Roman et al. (2012) proposed a long term exponential geometric distribution. The generalized exponential power series distributions have been proposed by Mahmoudi and Jafari (2012). An extended Weibull power series distribution has been proposed by Silva et al. (2013). Hassan et al. (2016) introduced the class of generalized inverse Weibull power series. Alkarni (2016) introduced generalized extended Weibull power series class.

Recently, compounding distributions for Lomax with discrete one have been presented by some authors. For instance; Abd-Elfattah et al. (2013), Ramos et al. (2013), Al-Zahrani and Sagor (2014), Al-Zahrani (2015), and Hassan and Abd-Alla (2017) have been proposed, respectively, Lomax Poisson, exponentiated Lomax Poisson, Lomax-Logarithmic, extended Poisson Lomax and exponentiated Lomax geometric distributions.

In this article, we proposed a new compound distribution by mixing the PL and Poisson distributions. The remainder of the article is organized as follows. Section 2 defines the subject distribution and gives the graphical presentation for the shape of its pdf and hazard rate function. Section 3 presents some structural properties of the new distribution such as; quantile, probability weighted moments, order statistics, entropy and moments of residual life. In Section 
4, the estimation of the model parameters is carried out using the maximum likelihood, least squares and weighted least squares methods. In Section 5, a simulation study is achieved to illustrate the theoretical results. Section 6 gives the applicability of the proposed model and compares with other competing probability models. At the end, concluding remakes are presented in Section 7.

\section{Power Lomax-Poisson Model}

In this section, we introduce and study the power Lomax Poisson (PLP) distribution. The probability density, cumulative distribution, reliability and hazard rate functions are obtained. Suppose that $X=\min \left\{Y_{i}\right\}_{i=1}^{Z}$ be independently and identically distributed (iid) failure times of $Z$ component connected in series and each $Y$ has the power Lomax distribution with pdf (3) and cdf (4). Let the random variable $Z$ has zero-truncated Poisson distribution with probability mass function given by

$$
P(Z=z)=\frac{e^{-\theta} \theta^{z}}{z !\left(1-e^{-\theta}\right)} ; z=1,2, \ldots, \quad \theta>0 .
$$

Assume that the variables $X$ 's and $Z$ are independent, then the conditional density function of $X \mid Z=Z$ is given by

$$
f_{X \mid Z}(x \mid z)=z \alpha \beta \lambda^{\alpha z} x^{\beta-1}\left(\lambda+x^{\beta}\right)^{-(\alpha z+1)} ; \quad x, \alpha, \lambda, \beta>0 .
$$

The joint distribution of the random variables $X$ and $Z$, denoted by $f_{X Z}(x ; z)$ is given by

$$
f_{X Z}(x ; z)=\frac{e^{-\theta} \theta^{z}}{z-1 !\left(1-e^{-\theta}\right)} \alpha \beta \lambda^{\alpha z} x^{\beta-1}\left(\lambda+x^{\beta}\right)^{-(\alpha z+1)} .
$$

The marginal pdf of $X$ is as follows

$$
f(x ; \Phi)=\alpha \beta \theta \lambda^{\alpha}\left(1-e^{-\theta}\right)^{-1} x^{\beta-1}\left(\lambda+x^{\beta}\right)^{-\alpha-1} e^{-\theta\left[1-\lambda^{\alpha}\left(\lambda+x^{\beta}\right)^{-\alpha}\right]} ; x, \alpha, \beta, \theta, \lambda>0,
$$

which defines the PLP distribution, where $\Phi \equiv(\alpha, \beta, \theta, \lambda)$. Or it can be written as follows

$f(x ; \Phi)=\alpha \beta \theta \lambda^{\alpha}\left(e^{\theta}-1\right)^{-1} x^{\beta-1}\left(\lambda+x^{\beta}\right)^{-\alpha-1} e^{\theta \lambda^{\alpha}\left(\lambda+x^{\beta}\right)^{-\alpha}} ; x, \alpha, \beta, \theta, \lambda>0$.

The distribution function of PLP is as follows

$$
F(x ; \Phi)=\frac{1-e^{-\theta\left[1-\lambda^{\alpha}\left(\lambda+x^{\beta}\right)^{-\alpha}\right]}}{\left(1-e^{-\theta}\right)} .
$$

Or it can be written as follows

$$
F(x ; \Phi)=\frac{e^{\theta}-e^{\theta \lambda^{\alpha}\left(\lambda+x^{\beta}\right)^{-\alpha}}}{\left(e^{\theta}-1\right)} .
$$

A random variable $X$ with density function (5) will be denoted by $X \sim P L P(\alpha, \beta, \theta, \lambda)$. 


\section{Lemma}

The power Lomax Poisson distribution reduces to the power Lomax distribution as $\theta \rightarrow 0$.

\section{Proof}

If $\theta$ approaches zero, then

$$
\lim _{\theta \rightarrow 0} F(x ; \Phi)=\frac{e^{\theta}-e^{\theta \lambda^{\alpha}\left(\lambda+x^{\beta}\right)^{-\alpha}\left[\lambda^{\alpha}\left(\lambda+x^{\beta}\right)^{-\alpha}\right]}}{e^{\theta}}=\left[1-\lambda^{\alpha}\left(\lambda+x^{\beta}\right)^{-\alpha}\right],
$$

which is the cdf of power Lomax distribution as defined in (4).

Furthermore, the reliability and hazard rate functions are as follows

and,

$$
R(x ; \Phi)=\frac{e^{\theta \lambda^{\alpha}\left(\lambda+x^{\beta}\right)^{-\alpha}}-1}{e^{\theta}-1},
$$

$$
h(x ; \Phi)=\frac{\alpha \beta \theta \lambda^{\alpha-1} x^{\beta-1}\left(\lambda+x^{\beta}\right)^{-\alpha-1} e^{\theta \lambda^{\alpha}\left(\lambda+x^{\beta}\right)^{-\alpha}}}{e^{\theta \lambda^{\alpha}\left(\lambda+x^{\beta}\right)^{-\alpha}}-1} .
$$

Figures 1 and 2 illustrate plots of the PLP densities and hazard rate functions for some selected values of the parameters.

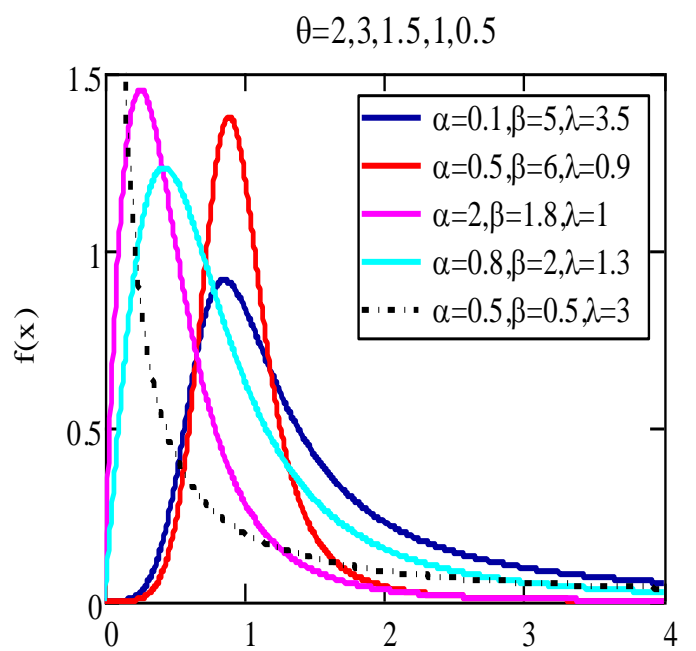

Figure 1: Pdfs of the PLP distribution for some parameter values

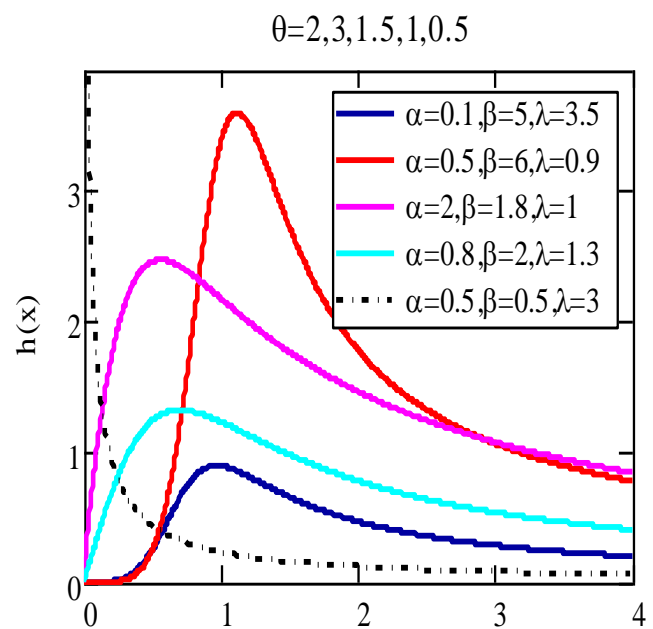

Figure 2: Hazard rate function of the PLP distribution for some parameter values 
Figure 1 shows that the density of PLP takes different shapes as symmetrical, right skewed, reversed-J and unimodel. From Figure 2, it can be observe that the shapes of the hazard rate function are increasing, decreasing and constant at some selected values of parameters.

\subsection{Some Structural Properties}

Here, some statistical properties of the PLP distribution including, quantile function, $r$ th moment, Re'nyi entropy, order statistics and moments of the residual life are obtained.

\subsection{Quantile Function}

The quantile function of PLP distribution, denoted by, $Q(u)=F^{-1}(u)$ of $X$ has the following form

$$
Q(u)=\left\{\lambda\left(\left[\frac{1}{\theta} \ln \left(e^{\theta}-u\left(e^{\theta}-1\right)\right)\right]^{\frac{-1}{\alpha}}-1\right)\right\}^{\frac{1}{\beta}},
$$

where $u$ is a uniform random variable on the unit interval $(0,1)$. In particular the median of the PLP distribution, denoted by $m$, is obtained by substituting $u=0.5$ in (7) as follows

$$
m=\left\{\lambda\left(\left[\frac{1}{\theta} \ln \left(0.5\left(e^{\theta}+1\right)\right)\right]^{\frac{-1}{\alpha}}-1\right)\right\}^{\frac{1}{\beta}} .
$$

\subsection{Moments}

Many of the important characteristics and features of a distribution can be obtained using ordinary moments. The $r$ th moment of $X$ can be easily obtained from pdf (5) as follows

$$
E\left(X^{r}\right)=\frac{\alpha \beta \theta \lambda^{\alpha}}{\left(e^{\theta}-1\right)} \int_{0}^{\infty} x^{r+\beta-1}\left(\lambda+x^{\beta}\right)^{-(\alpha+1)} e^{\theta \lambda^{\alpha}\left(\lambda+x^{\beta}\right)^{-\alpha}} d x .
$$

Using the exponential expansion for $e^{\theta \lambda^{\alpha}\left(\lambda+x^{\beta}\right)^{-\alpha}}$, then (8) can be written as follows

$$
E\left(X^{r}\right)=\sum_{j=0}^{\infty} \frac{\alpha \beta \theta^{j+1}}{j !\left(e^{\theta}-1\right) \lambda} \int_{0}^{\infty} x^{r+\beta-1}\left(1+\frac{x^{\beta}}{\lambda}\right)^{-(\alpha+\alpha j+1)} d x
$$

After simplification, the $r$ th moment of PLP distribution takes the following form

$$
E\left(X^{r}\right)=\sum_{j=0}^{\infty} \frac{\alpha \theta^{j+1} \lambda^{\frac{r}{\beta}}}{j !\left(e^{\theta}-1\right)} \frac{\Gamma\left(\frac{r}{\beta}+1\right) \Gamma\left(\alpha+\alpha j-\frac{r}{\beta}\right)}{\Gamma(\alpha+\alpha j+1)}, r=1,2, \ldots
$$

where $\Gamma($.$) stands for gamma function.$

In particular, the mean and variance of PLP distribution are obtained, respectively, as follows 


$$
\begin{gathered}
E(X)=\sum_{j=0}^{\infty} \frac{\alpha \theta^{j+1} \lambda^{\frac{1}{\beta}}}{j !\left(e^{\theta}-1\right)} \frac{\Gamma\left(\frac{1}{\beta}+1\right) \Gamma\left(\alpha+\alpha j-\frac{1}{\beta}\right)}{\Gamma(\alpha+\alpha j+1)}, \\
\operatorname{Var}(X)=\sum_{j=0}^{\infty} \frac{\alpha \theta^{j+1} \lambda^{\frac{2}{\beta}}}{j !\left(e^{\theta}-1\right)} \frac{\Gamma\left(\frac{2}{\beta}+1\right) \Gamma\left(\alpha+\alpha j-\frac{2}{\beta}\right)}{\Gamma(\alpha+\alpha j+1)}-\left[\sum_{j=0}^{\infty} \frac{\alpha \theta^{j+1} \lambda^{\frac{1}{\beta}}}{j !\left(e^{\theta}-1\right)} \frac{\Gamma\left(\frac{1}{\beta}+1\right) \Gamma\left(\alpha+\alpha j-\frac{1}{\beta}\right)}{\Gamma(\alpha+\alpha j+1)}\right]^{2} .
\end{gathered}
$$

Furthermore, the moment generating function can be obtained from moments as follows,

$$
M_{x}(t)=\sum_{r=0}^{\infty} \frac{t^{r}}{r !} \mu_{r}^{\prime}=\sum_{r, j=0}^{\infty} \frac{\alpha \theta^{j+1} \lambda^{\frac{r}{\beta}} t^{r}}{j ! r !\left(e^{\theta}-1\right)} \frac{\Gamma\left(\frac{r}{\beta}+1\right) \Gamma\left(\alpha+\alpha j-\frac{r}{\beta}\right)}{\Gamma(\alpha+\alpha j+1)},
$$

where, $\mu_{r}$ is the $r$ th moment about the origin.

\subsection{Re'nyi Entropy}

The entropy of a random variable $X$ is a measure of uncertainty variation. If $X$ is a random variable distributed as PLP, then the Re'nyi entropy, for $\rho>0$ and $\rho \neq 1$ is defined by:

$$
I_{\rho}(X)=\frac{1}{1-\rho} \log \int_{-\infty}^{\infty} f(x)^{\rho} d x, \quad \rho>0 \text { and } \rho \neq 1 .
$$

Then by using pdf (5), the Re'nyi entropy of PLP distribution can be written as follows:

$$
I_{\rho}(X)=\frac{1}{1-\rho} \log \left[\frac{\left(\alpha \beta \theta \lambda^{\alpha}\right)^{\rho}}{\left(e^{\theta}-1\right)^{\rho}} \int_{0}^{\infty} x^{\rho(\beta-1)}\left(\lambda+x^{\beta}\right)^{-\rho(\alpha+1)} e^{\rho\left(\theta \lambda^{\alpha}\left(\lambda+x^{\beta}\right)^{-\alpha}\right)} d x .\right]
$$

Using the exponential expansion and after simplification, then the Renyi entropy of PLP distribution takes the following form

$$
I_{\rho}(X)=\frac{1}{1-\rho} \log \left[\frac{\alpha^{\rho} \lambda^{\frac{1-\rho}{\beta}}}{\left(e^{\theta}-1\right)^{\rho} \beta^{1-\rho}} \sum_{j=0}^{\infty} \frac{\rho^{j} \theta^{\rho+j}}{j !} \mathrm{B}\left(\frac{\rho(\beta-1)+1}{\beta}, \rho(\alpha+1)+\alpha j-\frac{\rho(\beta-1)+1}{\beta}\right)\right],
$$

where, $\mathrm{B}(.,$.$) ) stands for beta function.$

\subsection{Moments of the Residual Life}

Several functions, namely; the failure rate function, mean residual life function and the left censored mean function are related to the residual life. These three functions uniquely determine $F(x)$. The $n$th moment of residual life denoted by $m_{n}(t)=E\left[(X-t)_{n} \mid X>\right.$ $t], n=1,2,3, \ldots$, is derived. The $n$th moment of the residual life of a random variable is defined as follows 


$$
m_{n}(t)=\frac{1}{R(t ; \Phi)} \int_{t}^{\infty}(x-t)^{n} f(x) d x
$$

The $n$th moment of the residual life of PLP distribution is obtained by inserting the pdf (5) and binomial expansion in $m_{n}(t)$ as follows

$$
m_{n}(t)=\frac{1}{R(t ; \Phi)} \sum_{r=0}^{n}(-1)^{n-r}\left(\begin{array}{l}
n \\
r
\end{array}\right) t^{n-r} \frac{\alpha \beta \theta \lambda^{\alpha}}{\left(e^{\theta}-1\right)} \int_{t}^{\infty} x^{r+\beta-1}\left(\lambda+x^{\beta}\right)^{-(\alpha+1)} e^{\theta \lambda^{\alpha}\left(\lambda+x^{\beta}\right)^{-\alpha}} d x .
$$

By using the exponential expansion, then $m_{n}(t)$ takes the following form

$$
m_{n}(t)=\frac{1}{R(t ; \Phi)} \sum_{r=0}^{n} \sum_{j=0}^{\infty}(-1)^{n-r}\left(\begin{array}{l}
n \\
r
\end{array}\right) \frac{t^{n-r} \alpha \beta \theta^{j+1}}{j !\left(e^{\theta}-1\right) \lambda} \int_{t}^{\infty} x^{r+\beta-1}\left(1+\frac{x^{\beta}}{\lambda}\right)^{-(\alpha+\alpha j+1)} d x .
$$

Put $z=\left(1+\frac{x^{\beta}}{\lambda}\right)^{-1}$, then the $n$th moment of the PLP distribution takes the following form

$$
m_{n}(t)=\frac{1}{\left(e^{\theta \lambda^{\alpha}\left(\lambda+t^{\beta}\right)^{-\alpha}}-1\right)} \sum_{r=0}^{n} \sum_{j=0}^{\infty}(-1)^{n-r}\left(\begin{array}{l}
n \\
r
\end{array}\right) \frac{t^{n-r} \alpha \theta^{j+1} \lambda^{\frac{r}{\beta}}}{j !} \mathrm{B}\left(\left(1+\frac{t^{\beta}}{\lambda}\right)^{-1},\left(\frac{r}{\beta}+1\right),\left(\alpha+\alpha j-\frac{r}{\beta}\right)\right),
$$

which is incomplete beta. In particular, the mean residual life of the PLP distribution is obtained by substituting $n=1$ in (10) as follows

$$
m_{1}(t)=\frac{1}{\left(e^{\theta \lambda^{\alpha}\left(\lambda+t^{\beta}\right)^{-\alpha}}-1\right)} \sum_{j=0}^{\infty} \frac{\alpha \theta^{j+1} \lambda^{\frac{1}{\beta}}}{j !} \mathrm{B}\left(\left(1+\frac{t^{\beta}}{\lambda}\right)^{-1},\left(\frac{1}{\beta}+1\right),\left(\alpha+\alpha j-\frac{1}{\beta}\right)\right)-t .
$$

\subsection{Order Statistics}

Suppose $X_{1}, X_{2}, \ldots, X_{n}$ is a random sample from PLP distribution. Let $X_{1: n}, X_{2: n}, \ldots, X_{n: n}$ denote the corresponding order statistics. It is well known that the probability density function of the $k$ th order statistics is given by

$$
f_{k: n}(x)=\frac{f(x)}{B(k, n-k+1)} \sum_{u=0}^{n-k}(-1)^{u}\left(\begin{array}{c}
n-k \\
u
\end{array}\right) F(x)^{k+u-1},
$$

Inserting cdf (6) and pdf (5) in (11), then

$$
\begin{aligned}
f_{k: n}(x) & =\frac{\alpha \beta \theta \lambda^{\alpha} x^{\beta-1}}{B(k, n-k+1)}\left(\lambda+x^{\beta}\right)^{-(\alpha+1)} e^{\theta \lambda^{\alpha}\left(\lambda+x^{\beta}\right)^{-\alpha}} \sum_{u=0}^{n-k}(-1)^{u}\left(\begin{array}{c}
n-k \\
u
\end{array}\right)\left(e^{\theta}-1\right)^{-(u+k)} \\
& {\left[e^{\theta}-e^{\theta \lambda^{\alpha}\left(\lambda+x^{\beta}\right)^{-\alpha}}\right]^{u+k-1} }
\end{aligned}
$$

Using the power series for the exponential function will be

$$
e^{\theta \lambda^{\alpha}\left(\lambda+x^{\beta}\right)^{-\alpha}}=\sum_{q=0}^{\infty} \frac{\theta^{q} \lambda^{q \alpha}}{q !}\left(\lambda+x^{\beta}\right)^{-\alpha q} .
$$


Then, substituting (13) in (12), the pdf of the $k$ th order statistics takes the following form

$$
\begin{aligned}
f_{k: n}(x)= & \frac{\alpha \beta x^{\beta-1}}{B(k, n-k+1)} \sum_{u=0}^{n-k} \sum_{q=0}^{\infty}(-1)^{u}\left(\begin{array}{c}
n-k \\
u
\end{array}\right) \frac{\theta^{q+1}}{q ! \lambda\left(e^{\theta}-1\right)^{(u+k)}}\left(1+\frac{x^{\beta}}{\lambda}\right)^{-(q \alpha+\alpha+1)} \\
& \times\left[e^{\theta}-e^{\theta \lambda^{\alpha}\left(\lambda+x^{\beta}\right)^{-\alpha}}\right]^{u+k-1} .
\end{aligned}
$$

Hence, the pdf of the $k$ th order statistics of $X$ is as follows;

$$
f_{k: n}(x)=\alpha \beta x^{\beta-1} \sum_{u=0}^{n-k} \sum_{q=0}^{\infty} \eta_{k, u, q}\left(1+\frac{x^{\beta}}{\lambda}\right)^{-(q \alpha+\alpha+1)}\left[e^{\theta}-e^{\theta \lambda^{\alpha}\left(\lambda+x^{\beta}\right)^{-\alpha}}\right]^{u+k-1} \text {, }
$$

where,

$$
\eta_{k, u, q}=(-1)^{u}\left(\begin{array}{c}
n-k \\
u
\end{array}\right) \frac{\theta^{q+1}}{q ! \lambda\left(e^{\theta}-1\right)^{(u+k)} B(k, n-k+1)} .
$$

In particular, the pdf of the smallest order statistics $X_{1: n}$ is obtained from (14), by substituting $k=1$, as follows

$$
f_{1: n}(x)=\alpha \beta x^{\beta-1} \sum_{u=0}^{n-1} \sum_{q=0}^{\infty} \eta_{1, u, q}\left(1+\frac{x^{\beta}}{\lambda}\right)^{-(q \alpha+\alpha+1)}\left[e^{\theta}-e^{\theta \lambda^{\alpha}\left(\lambda+x^{\beta}\right)^{-\alpha}}\right]^{u},
$$

where,

$$
\eta_{1, u, q}=(-1)^{u}\left(\begin{array}{c}
n-1 \\
u
\end{array}\right) \frac{n \theta^{q+1}}{q ! \lambda\left(e^{\theta}-1\right)^{(u+1)}} .
$$

Also, the pdf of the largest order statistics $X_{n: n}$ is obtained from (14), by substituting $k=n$.

$$
f_{n: n}(x)=\alpha \beta x^{\beta-1} \sum_{q=0}^{\infty} \eta_{n, u, q}\left(1+\frac{x^{\beta}}{\lambda}\right)^{-(q \alpha+\alpha+1)}\left[e^{\theta}-e^{\theta \lambda^{\alpha}\left(\lambda+x^{\beta}\right)^{-\alpha}}\right]^{u},
$$

where,

$$
\eta_{n, u, q}=(-1)^{u} \frac{n \theta^{q+1}}{q ! \lambda\left(e^{\theta}-1\right)^{(u+n)}} .
$$

\section{Parameter Estimation}

In this section, the estimates of the PLP model parameters are obtained using maximum likelihood, least squares and weighted least squares methods.

\subsection{Maximum Likelihood Estimators}

Let $X_{1}, X_{2}, \ldots, X_{n}$ be a simple random sample from the PLP distribution with set of parameters $\Phi \equiv(\alpha, \lambda, \theta, \beta)$. The log-likelihood function, denoted by $\ln l$, based on the observed random sample of size $n$ from density (5) is given by: 
$\ln l=n \ln \alpha+n \ln \theta+n \ln \beta+n \alpha \ln \lambda-n \ln \left(e^{\theta}-1\right)+(\beta-1) \sum_{i=1}^{n} \ln x_{i}-(\alpha+1) \sum_{i=1}^{n} \ln S_{i}+\theta \lambda^{\alpha} \sum_{i=1}^{n} S_{i}^{-\alpha}$,

where $S_{i}=\left(\lambda+x_{i}\right)^{\beta}$. The partial derivatives of the log-likelihood function with respect to the unknown parameters are given by:

$$
\begin{gathered}
\frac{\partial \ln l}{\partial \alpha}=\frac{n}{\alpha}+n \ln \lambda-\sum_{i=1}^{n} \ln S_{i}+\theta \lambda^{\alpha} \sum_{i=1}^{n} S_{i}^{-\alpha}\left(\ln \lambda-\ln S_{i}\right), \\
\frac{\partial \ln l}{\partial \lambda}=\frac{n \alpha}{\lambda}-(\alpha+1) \sum_{i=1}^{n} \ln S_{i}^{-1}+\theta \alpha \lambda^{\alpha} \sum_{i=1}^{n} S_{i}^{-\alpha}\left(\lambda^{-1}-S_{i}^{-1}\right), \\
\frac{\partial \ln l}{\partial \theta}=\frac{n}{\theta}-n e^{\theta}\left(e^{\theta}-1\right)^{-1}+\lambda^{\alpha} \sum_{i=1}^{n} S_{i}^{-\alpha},
\end{gathered}
$$

and,

$$
\frac{\partial \ln l}{\partial \beta}=\frac{n}{\beta}+\sum_{i=1}^{n} \ln x_{i}-(\alpha+1) \sum_{i=1}^{n} \ln S_{i}^{-1} x_{i}^{\beta} \ln x_{i}-\theta \alpha \lambda^{\alpha} \sum_{i=1}^{n} S_{i}^{-(\alpha+1)} x_{i}^{\beta} \ln x_{i} .
$$

The maximum likelihood estimators (MLEs) of the model parameters are determined by solving numerically the non-linear equations $\frac{\partial \ln l}{\partial \alpha}=0, \frac{\partial \ln l}{\partial \lambda}=0, \frac{\partial \ln l}{\partial \theta}=0$ and $\frac{\partial \ln l}{\partial \beta}=0$ simultaneously.

\subsection{Least Squares and Weighted Least Squares Estimators}

Suppose $X_{1}, X_{2}, \ldots, X_{n}$ is a random sample of size $n$ from PLP distribution and suppose $X_{1: n}<X_{2: n}<\ldots<X_{n: n}$ denotes the corresponding ordered sample. According to Johnson et al. (1995), the expectation and the variance of distribution are independent of the unknown parameter and are given by

$$
E\left(F\left(X_{i: n}\right)\right)=\frac{i}{n+1}, \quad \text { and } \operatorname{Var}\left(F\left(X_{i: n}\right)\right)=\frac{i(n-i+1)}{(n+1)^{2}(n+2)},
$$

where $F\left(X_{i: n}\right)$ is cdf for any distribution and $X_{i: n}$ is the $i$ th order statistic. Then the least squares (LS) estimators can be obtained by minimizing the sum of squares errors

$$
\sum_{i=1}^{n}\left[F\left(X_{i: n}\right)-\frac{i}{n+1}\right]^{2}
$$

with respect to the unknown parameters. So the least squares estimators of the unknown parameters $\alpha, \lambda, \theta$ and $\beta$, denoted by $\ddot{\alpha}, \ddot{\lambda}, \ddot{\theta}$ and $\ddot{\beta}$, of the PLP model can be obtained by minimizing the following quantity

$$
\sum_{i=1}^{n}\left[\frac{e^{\theta}-e^{\theta \lambda^{\alpha}\left(\lambda+x_{i}^{\beta}\right)^{-\alpha}}}{e^{\theta}-1}-\frac{i}{n+1}\right]^{2}
$$

with respect to $\alpha, \lambda, \theta$ and $\beta$. 
Weighted least squares (WLS) estimators can be obtained by minimizing the sum of squares errors

$$
\sum_{i=1}^{n} \frac{1}{\operatorname{Var}\left(F\left(X_{i: n}\right)\right)}\left[F\left(X_{i: n}\right)-E\left(F\left(X_{i: n}\right)\right)\right]^{2}
$$

with respect to the unknown parameters $\alpha, \lambda, \theta$ and $\beta$. Therefore, the weighted least squares estimators $\dddot{\alpha}, \dddot{\lambda}, \dddot{\theta}$ and $\dddot{\beta}$ can be obtained by minimizing the following quantity

$$
\sum_{i=1}^{n} \frac{(n+1)^{2}(n+2)}{i(n-i+1)}\left[\frac{e^{\theta}-e^{\theta \lambda^{\alpha}\left(\lambda+x_{i}^{\beta}\right)^{-\alpha}}}{e^{\theta}-1}-\frac{i}{n+1}\right]^{2},
$$

with respect to the parameters $\alpha, \lambda, \theta$ and $\beta$.

\section{Simulation Study}

In any estimation problems, it is required to study the properties of the derived estimators. The derived expressions for the estimators are too complicated to study analytically. Consequently, a numerical study will be set up, treating separately the sampling distribution of the estimators. A numerical study is performed to compare the different estimators discussed in the previous section. The performances of the different estimators are compared in terms of their relative bias (RB), mean square error (MSE) and standard error (SE). The numerical procedures will be described below:

Step 1: 1000 random samples of size 10, 20, 30, 40, 50 and 100 are generated from the power Lomax Poisson distribution.

Step 2: Three sets of parameters values are selected as; case $I \equiv(\alpha=0.75, \lambda=0.5, \theta=1, \beta=$ $0.5)$, case II $\equiv(\alpha=0.5, \lambda=0.5, \theta=1, \beta=0.25)$ and case III $\equiv(\alpha=0.35, \lambda=0.5, \theta=$ $1, \beta=0.2)$.

Step 3: the MLEs, LS estimators and WLS estimators of the unknown parameters are obtained.

Step 4: The biases, MSEs and SEs of different estimators of unknown parameters are computed.

Simulation results are reported in Tables (1) and (2) (at the end of the article) and represented through some Figures from (3) to (10). From these tables and figures, the following conclusions can be observed on the performance of different estimators.

1. For all methods of estimations, it is clear that MSEs and SEs decrease as sample size increases (see Tables (1) and (2)).

2. The MSEs of MLEs, for all parameters values, are the smallest among the other estimators in almost all cases (see for example Table (1) and Figures (3-5)). 


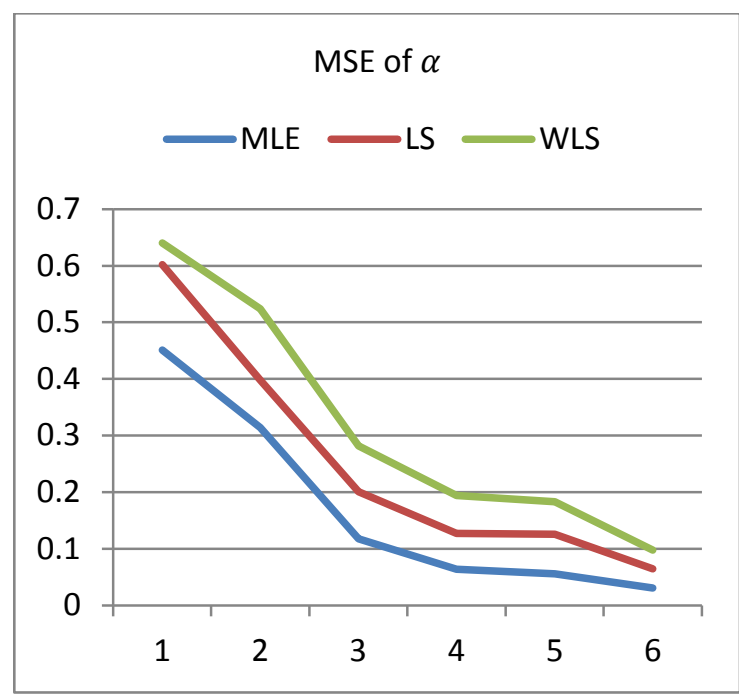

Figure 3: The MSE for $\alpha$ of the first case based on MLE, LS, WLS methods

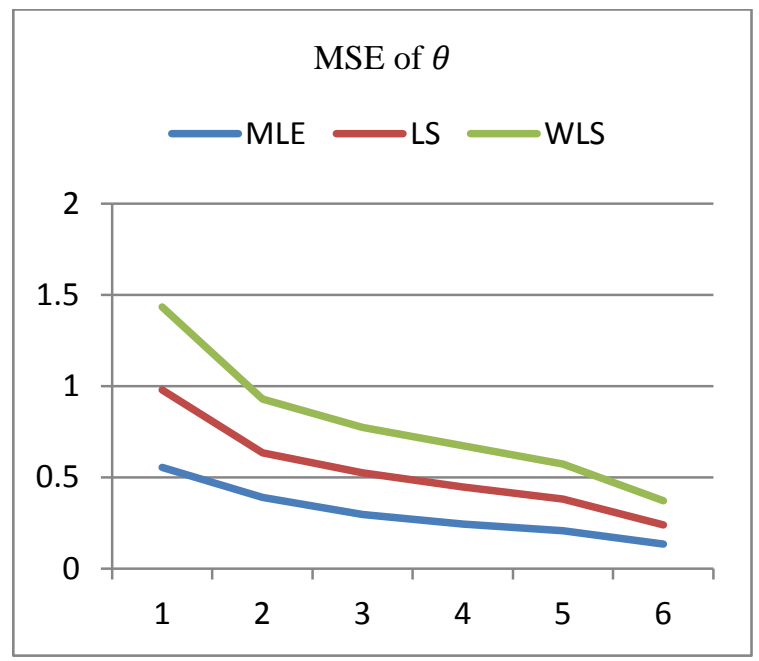

Figure 5: The MSE for $\theta$ of the second case based on MLE, LS, WLS methods

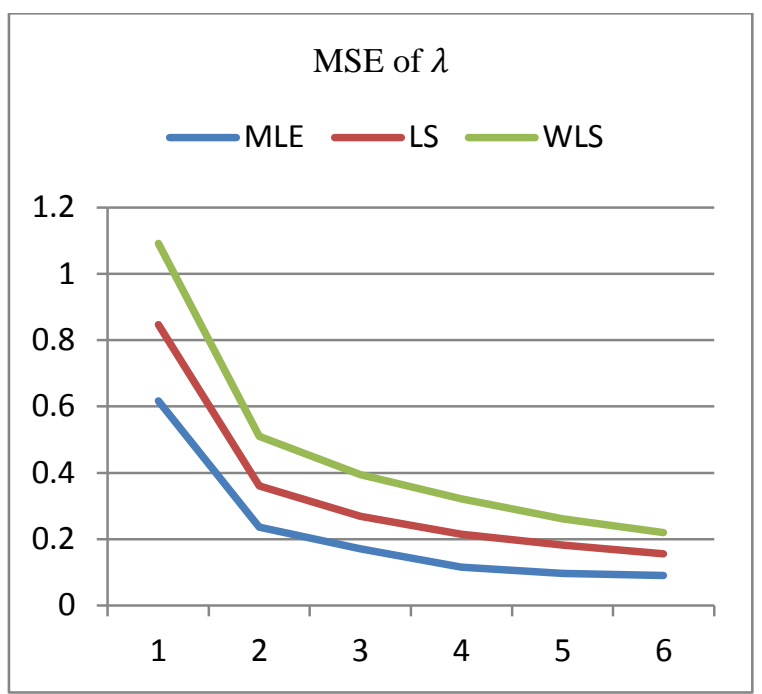

Figure 4: The MSE for $\lambda$ of the third case based on MLE, LS, WLS methods

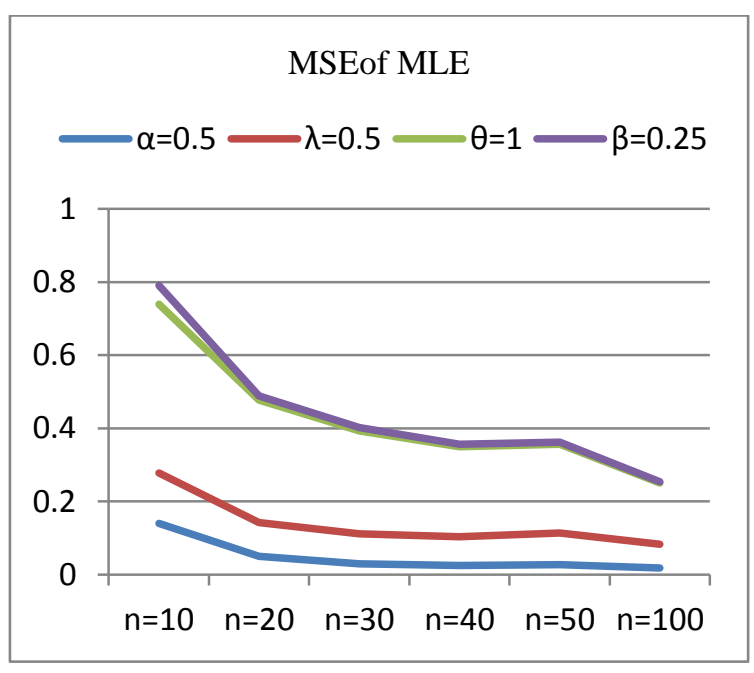

Figure 6: MSE for MLEs for the second case of parameters

3. The MSEs of MLEs $\hat{\alpha}, \hat{\lambda}, \hat{\theta}$ and $\hat{\beta}$ decrease as the sample size increases for different selected set of parameters (see for example Figure (6)) based on the case II. The MSEs of LS estimators $\ddot{\alpha}, \ddot{\lambda}, \ddot{\theta}$ and $\ddot{\beta}$ decrease for different selected set of parameters as the sample size increases based on case III (see for example Figure (7)).

4. The MSEs of the WLS estimators $\dddot{\alpha}, \dddot{\lambda}, \dddot{\theta}$ and $\dddot{\beta}$ decrease as the sample sizes increase for different selected set of parameters (see for example Figure (8)). 


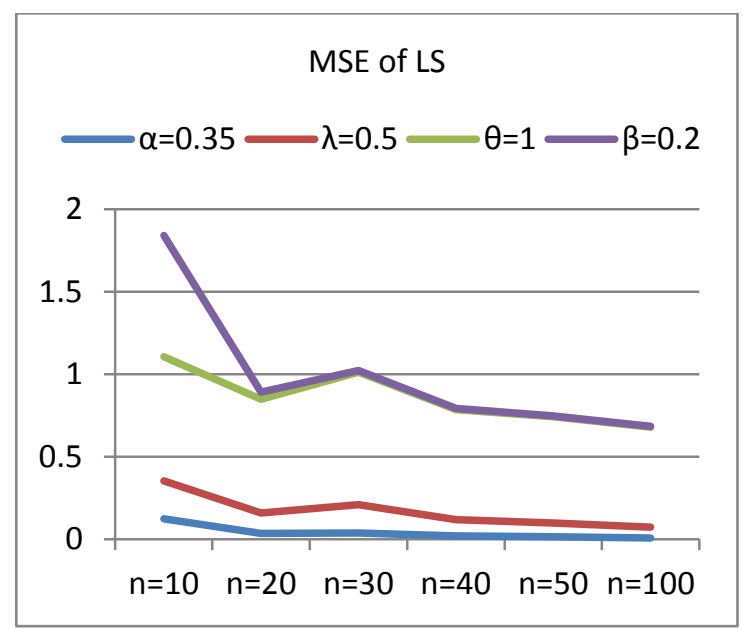

Figure 7: MSE for LSs for the third case of parameters

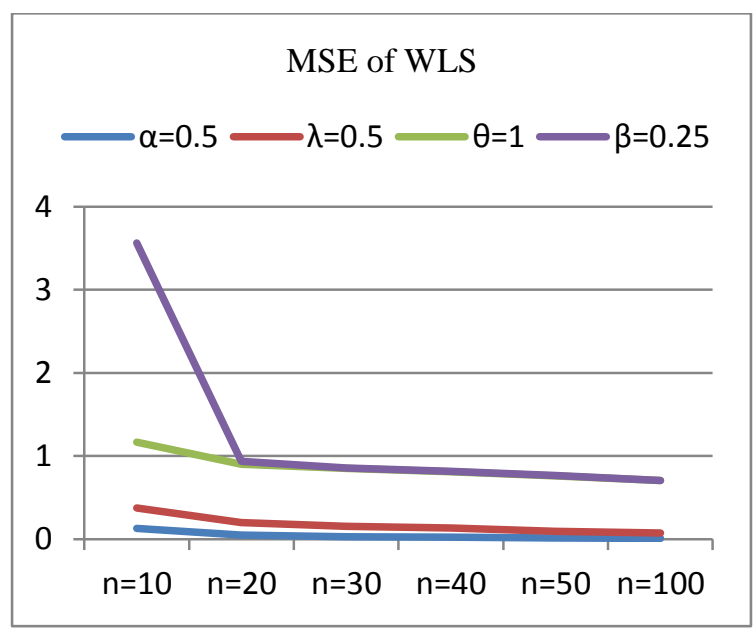

Figure 8: MSE for WLSs for the second case of parameters

5. The MSEs of the MLEs of $\alpha$ are smaller than the corresponding MSEs for the other methods in almost all the cases (see for example Figures $(9 a, b)$ ). Also, the MSEs of the MLEs of $\alpha$ for the case I have the smallest values corresponding to the MSEs for the other sets of parameters for the same sample size (see Figure ( 9 a)). The MSEs of MLEs of $\alpha$ for the case I have the smallest values corresponding to MSEs of the other cases of parameters for the same sample size (see Figure $(9 \mathrm{~b})$ ).

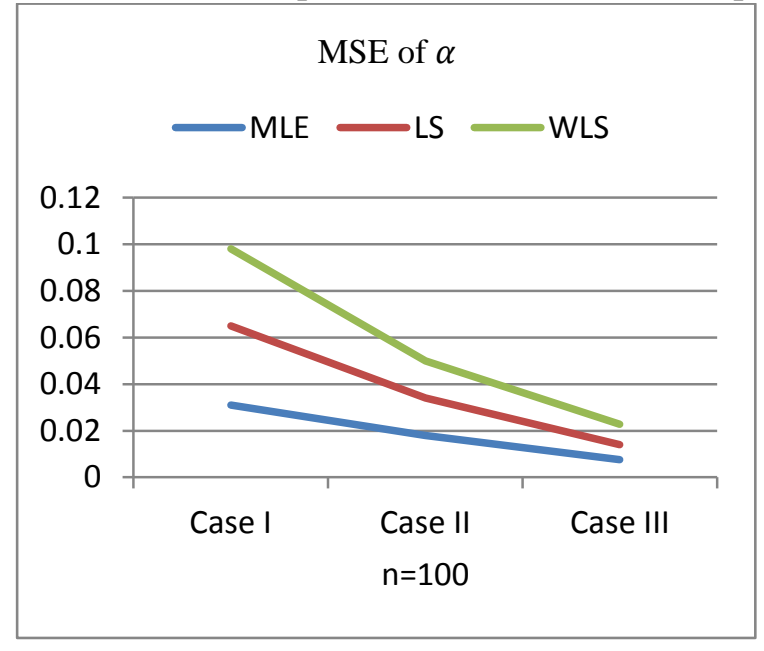

Figure 9a: MSEs of $\alpha$ for all the cases based on different methods

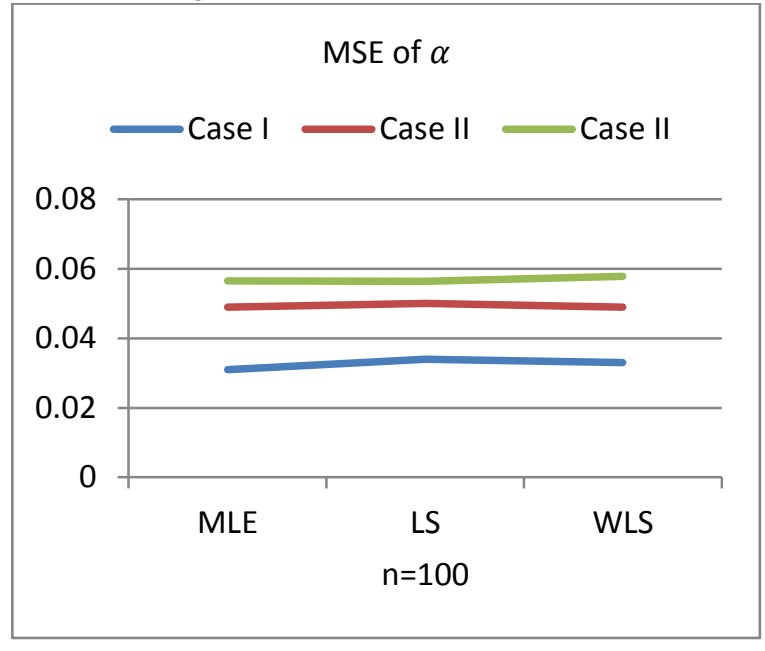

Figure 9b: MSEs of $\alpha$ for all the cases based on different methods

6. As it seems from Figures (10 a, b), the MSEs of the MLEs of $\beta$ take the smallest values corresponding to the MSEs of the other estimators $\ddot{\beta}$ and $\dddot{\beta}$ for the same sample size. Also, the MSEs of the MLEs of $\beta$ for case I have the smallest values corresponding to MSEs of the two other cases for the same sample size (see Figure 
(10 a)). The MSEs of MLEs of $\beta$ for the case I have the smallest values corresponding to MSEs of the other cases for the same sample size (see Figure $(10 \mathrm{~b})$ ).

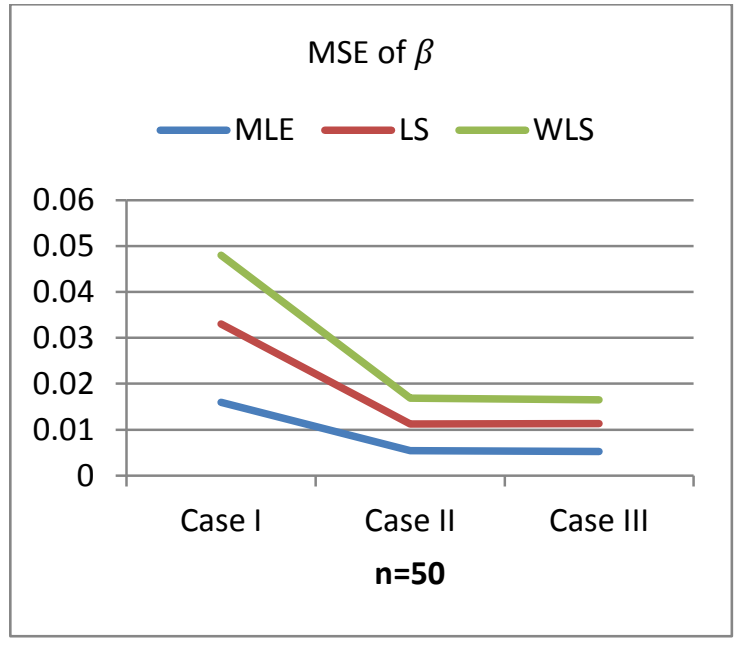

Figure 10 a: MSEs of the estimate $\beta$ for all the cases based on different methods

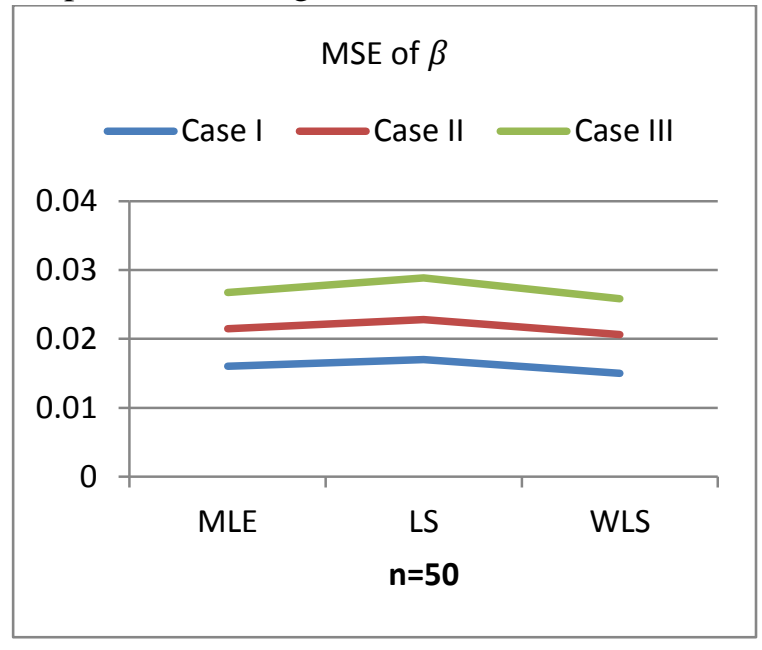

Figure $10 \mathrm{~b}$ : MSEs of the estimate $\beta$ for all the cases based on different methods

7. For fixed value of $(\lambda=0.5, \theta=1)$ and as the value of shape parameters $(\alpha, \beta)$ increases, the MSEs for estimators based on maximum likelihood, least squares and weighted least squares methods are increasing (see Table(1)).

8. For fixed value of $(\lambda=0.5, \theta=1)$ and as the value of shape parameters $(\alpha, \beta)$ decrease, the MSEs for estimators based on maximum likelihood, least squares and weighted least squares methods are decreasing (see Table(1)).

9. The MSEs of $\lambda$ for LS estimators and WLS estimators are approximately constant, when the shape parameters $(\alpha, \beta)$ increase.

\section{Applications To Real Data}

In this section, an application of the proposed PLP model to two real data sets is provided to show the flexibility and applicability of the new model in practice. For the first data, the PLP distribution is compared with Kumuerswmay Lomax (KL), power Lomax, beta Lomax (BL), exponentiated Lomax (EL) and Lomax (L) distributions. While, for the second data, the PLP distribution is compared with Weibull Lomax (WL), KL, BL, EL and L distributions

Example 6.1: The first data set represents 84 observations of failure time for particular windshield model given in Table 16.11 of Murthy et al. (2004). The data are recorded as follows:

$0.040,1.866,2.385,3.443,0.301,1.876,2.481,3.467,0.309,1.899,2.610,3.478,0.557,1.911$, $2.625,3.578,0.943,1.912,2.632,3.595,1.070,1.914,2.646,3.699,1.124,1.981,2.661$, $3.779,1.248,2.010,2.688,3.924,1.281,2.038,2.82,3,4.035,1.281,2.085,2.890,4.121,1.303$, $2.089,2.902,4.167,1.432,2.097,2.934,4.240,1.480,2.135,2.962,4.255,1.505,2.154,2.964$, 4.278, 1.506, 2.190, 3.000, 4.305, 1.568, 2.194, 3.103, 4.376, 1.615, 2.223, 3.114, 4.449, 1.619, 
$2.224,3.117,4.485,1.652,2.229,3.166,4.570,1.652,2.300,3.344,4.602,1.757,2.324,3.376$, 4.663.

To compare the fitted models, some criteria measures like; Akaike information criterion (AIC), Bayesian information criterion (BIC), consistent Akaike information criterion (CAIC), Hannan-Quinn information criterion (HQIC) and the Kolmogorov-Smirnov (K-S) statistics are considered. Generally, the smaller values of these statistics are corresponding to the better fit model to the data. The mathematical form of these measures is as follows

$$
\begin{gathered}
A I C=2 k-2 \ln l, B I C=k \ln (n)-2 \ln l, C A I C=A I C+\frac{2 k(k+1)}{n-k-1}, \\
H Q I C=2 k \ln [\ln (n)]-2 \ln l, \quad k-S=\sup _{y}\left[F_{n}(\mathrm{y})-F(\mathrm{y})\right],
\end{gathered}
$$

where $k$ is the number of models parameter, $n$ is the sample size and $\ln l$ is the maximized value of the log- likelihood function under the fitted models. Table 3 lists the numerical values of the statistics measures.

Table 3: Statistics measures for all models for failure times of 84Aircraff Windshield data

\begin{tabular}{|c|c|c|c|c|c|c|}
\hline \multirow{2}{*}{ Models } & \multicolumn{7}{|c|}{ Statistics } \\
\cline { 2 - 7 } & $\mathbf{- 2 l o g} l$ & AIC & CAIC & BIC & HQIC & K-S \\
\hline PLP & $\mathbf{2 6 1 . 7 3 6}$ & $\mathbf{2 6 9 . 7 3 6}$ & $\mathbf{2 7 0 . 2 4 3}$ & $\mathbf{2 7 9 . 4 6 0}$ & $\mathbf{2 7 3 . 6 4 5}$ & $\mathbf{0 . 0 9 6}$ \\
\hline KL & $\mathbf{2 6 4 . 8 0 8}$ & $\mathbf{2 7 2 . 8 0 9}$ & $\mathbf{2 7 3 . 3 0 9}$ & $\mathbf{2 8 2 . 5 8 0}$ & $\mathbf{2 7 6 . 7 3 9}$ & $\mathbf{0 . 0 9 7}$ \\
\hline BL & $\mathbf{2 7 7 . 4 3 4}$ & $\mathbf{2 8 5 . 4 3 5}$ & $\mathbf{2 8 5 . 9 3 5}$ & $\mathbf{2 9 5 . 2 0 6}$ & $\mathbf{2 8 9 . 3 6 5}$ & $\mathbf{0 . 1 9 3}$ \\
\hline EL & $\mathbf{2 8 2 . 7 9 9}$ & $\mathbf{2 8 8 . 7 9 9}$ & $\mathbf{2 8 9 . 0 9 5}$ & $\mathbf{2 9 6 . 1 2 7}$ & $\mathbf{9 2 1 . 7 4 6}$ & $\mathbf{0 . 8 3 5}$ \\
\hline PL & $\mathbf{4 4 0 . 2 6 7}$ & $\mathbf{4 4 6 . 2 6 7}$ & $\mathbf{4 4 6 . 5 6 0}$ & $\mathbf{4 5 3 . 5 6 0}$ & $\mathbf{4 4 9 . 1 9 9}$ & $\mathbf{0 . 9 9 9}$ \\
\hline L & $\mathbf{3 2 9 . 9 7 7}$ & $\mathbf{3 3 3 . 9 7 7}$ & $\mathbf{3 3 4 . 1 2 3}$ & $\mathbf{3 3 8 . 8 6 2}$ & $\mathbf{3 3 5 . 9 4 2}$ & $\mathbf{1}$ \\
\hline
\end{tabular}

It is clear from Table 3 that the PLP model has the smallest values for the statistics measures among all fitted models. So, the PLP model could be chosen as the best model. Further, figures of the estimated cumulative and estimated densities of the fitted models are presented in Figures 11 and 12 


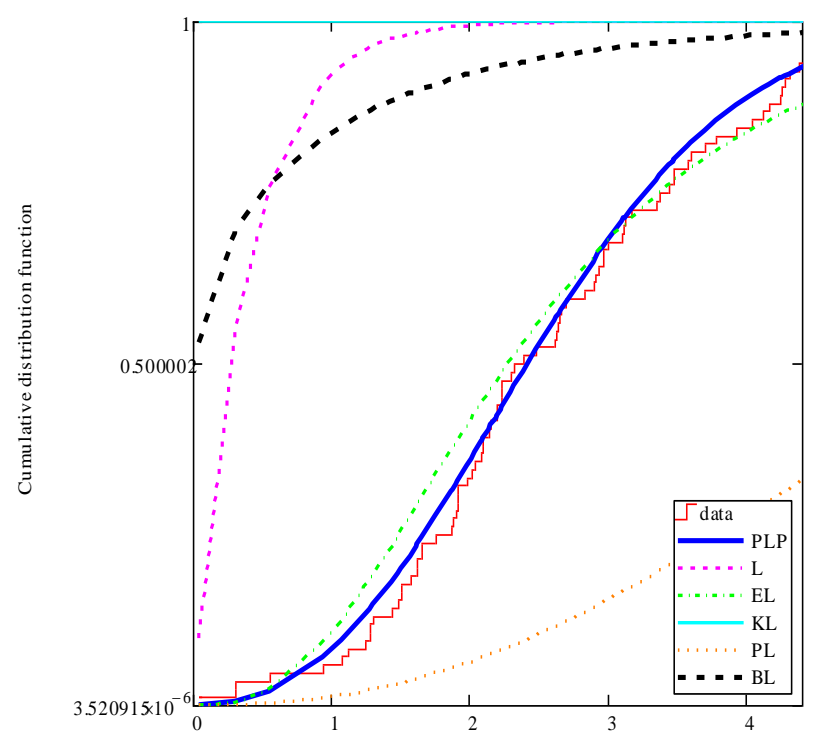

Figure. 11 Estimated cumulative densities of models for the first data set

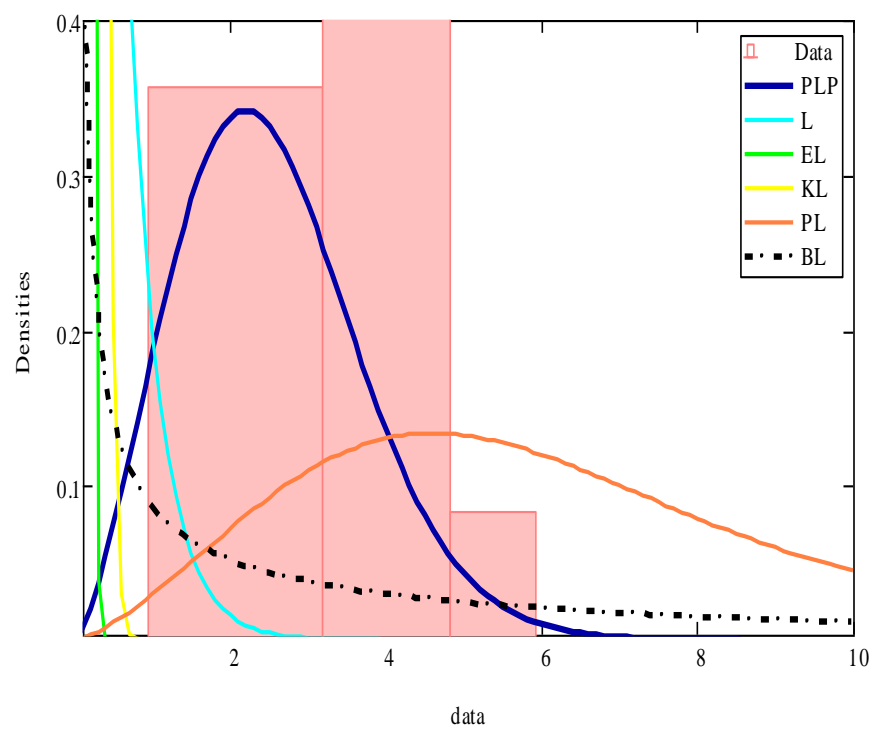

Figure 12. Estimated densities of models for the first data set

As seen from the above two figures that, the PLP distribution provides a closer fit to the histogram and then it is the best model among the other models to analyze these data.

Example 6.2: The second data set represents the survival times (in days) of 72 guinea pigs infected with virulent tubercle bacilli, observed and reported by Bjerkedal (1960). The data are as follows: 
$0.1,0.33,0.44,0.56,0.59,0.59,0.72,0.74,0.92,0.93,0.96,1,1,1.02,1.05,1.07,1.07,1.08$, $1.08,1.08,1.09,1.12,1.13,1.15,1.16,1.2,1.21,1.22,1.22,1.24,1.3,1.34,1.36,1.39,1.44$, $1.46,1.53,1.59,1.6,1.63,1.68,1.71,1.72,1.76,1.83,1.95,1.96,1.97,2.02,2.13,2.15,2.16$, $2.22,2.3,2.31,2.4,2.45,2.51,2.53,2.54,2.78,2.93,3.27,3.42,3.47,3.61,4.02,4.32,4.58$, $5.55,2.54,0.77$.

Table 4 lists the numerical values of the statistics measures for the second data set, plots of the estimated cumulative and estimated densities of the fitted models are displayed in Figures 13 and 14.

Tables 4: Statistics measures for all models based on the second data set

\begin{tabular}{|c|c|c|c|c|c|c|}
\hline \multirow{2}{*}{ Models } & \multicolumn{7}{|c|}{ Statistics } \\
\cline { 2 - 7 } & $-2 \log l$ & AIC & CAIC & BIC & HQIC & K-S \\
\hline PLP & $\mathbf{1 8 7 . 1 8 1}$ & 195.181 & 195.778 & 204.740 & 198.807 & $\mathbf{0 . 0 7 8}$ \\
\hline KL & 190.254 & 198.254 & 198.835 & 207.361 & 201.879 & $\mathbf{0 . 0 9 9}$ \\
\hline BL & 205.238 & 213.238 & 216.863 & 213.835 & 216.863 & 0.126 \\
\hline WL & 189.198 & 197.148 & 206.255 & 197.728 & 200.774 & 0.098 \\
\hline EL & $\mathbf{2 4 5 . 3 2 6}$ & $\mathbf{2 5 1 . 3 2 6}$ & $\mathbf{2 5 8 . 1 5 6}$ & $\mathbf{2 5 1 . 6 6 9}$ & $\mathbf{2 5 4 . 0 4 5}$ & $\mathbf{0 . 2 6 1}$ \\
\hline L & $\mathbf{2 2 6 . 1 5 3}$ & $\mathbf{2 3 0 . 1 5 3}$ & $\mathbf{1 9 7 . 7 7 8}$ & $\mathbf{2 0 6 . 2 8 8}$ & $\mathbf{2 0 0 . 7 7 4}$ & $\mathbf{0 . 2 9 5}$ \\
\hline
\end{tabular}

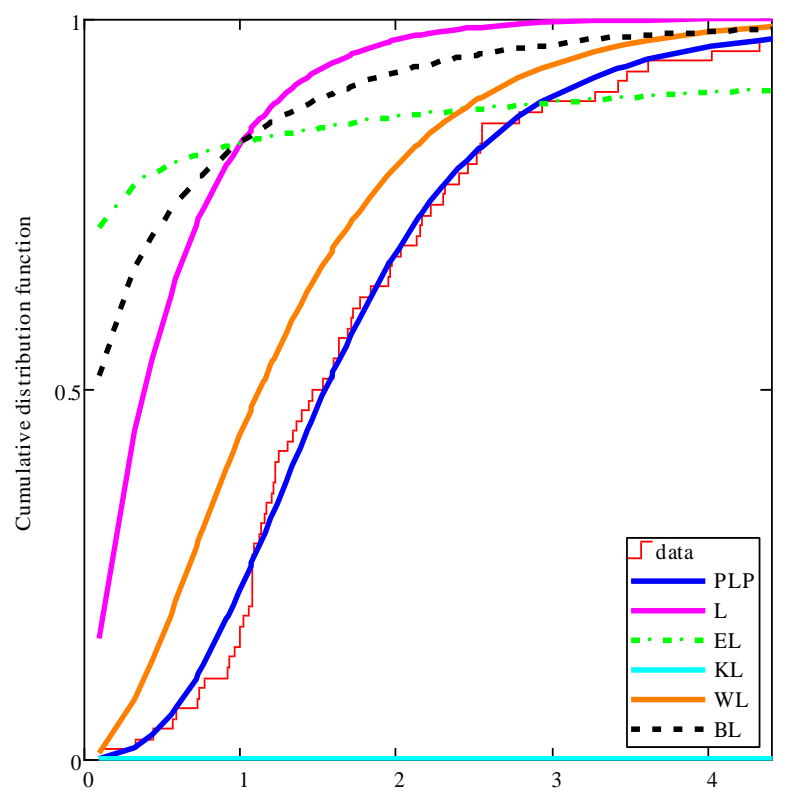

Figure13: Estimated cumulative densities of models for the second data set 


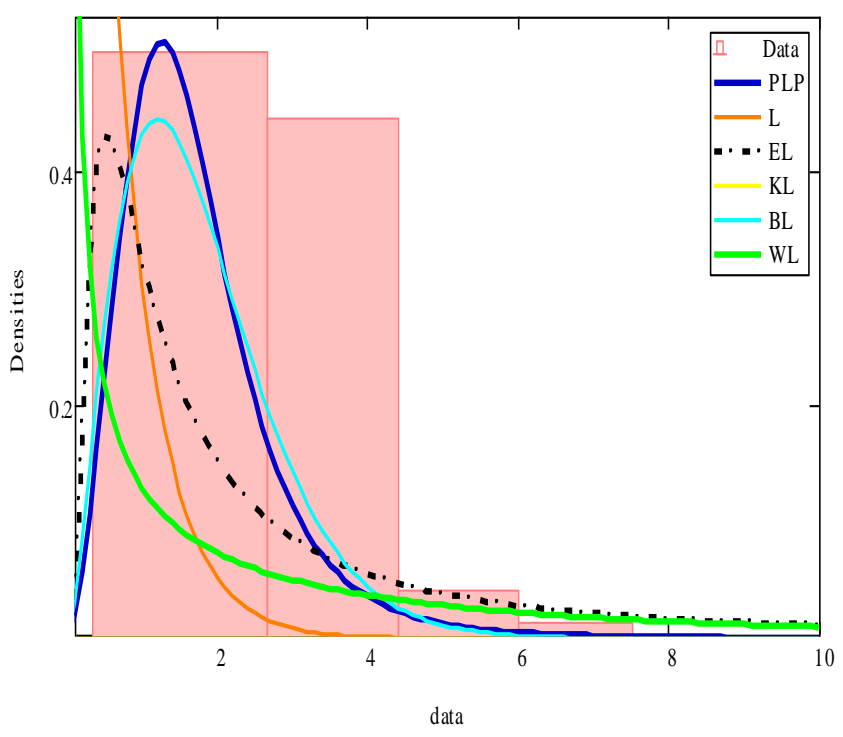

Figure 14: Estimated densities of models for the second data set

The values in Table 4 indicate that the most fitted distribution to the data is PLP distribution compared to other distributions. Also, it gives the best fit to these data.

\section{Concluding Remarks}

In this paper we have introduced a new four- parameter compounding distribution, called the power Lomax Poisson distribution. Statistical properties of the new distribution such as, moments, mean residual life, order statistics, quantile measures and Re'nyi entropy are obtained. Three methods of estimation, namely; maximum likelihood, least squares, and weighted least squares are proposed to estimate the model parameters and simulation results are provided to assess the model performance. The PLP model is fitted to two real life data sets to illustrate the usefulness of the proposed distribution. The new model provides consistently a better fit than the other competitive models. 


\section{References}

[1] Abdul-Moniem, I. B. and Abdel-Hameed, H. F. (2012). A lifetime distribution with decreasing failure rate. International Journal of Mathematical Education, 33 (5), 1-7.

[2] Abd-Elfattah, A. M, Hassan, A. S. and Hussein, A. M. (2013). On the Lomax -Poisson Distribution. Proceeding of the 48th The Annual Conference On Statistics, Computer Sciences \& Operations Research, Institute of Statistical Studies Research, Cairo University, 25-39.

[3] Adamidis K., and Loukas, S. (1998). A lifetime distribution with decreasing failure rate. Statistics and Probability Letters, 39, 35-42.

[4] Alkarni, S. H. (2016). Generalized extended Weibull power series family of distributions. Journal of Data Science, 14 (3), 415-439.

[5] Al-Zahrani, B. (2015). An extended Poisson-Lomax distribution. Advances in Mathematics: Scientific Journal, 4(2), 79-89.

[6] Al-Zahrani, B. and Sagor, H. (2014). Statistical analysis of the Lomax-Logarithmic distribution. Journal of Statistical Computation and Simulation, 85, 1883-1901.

[7] Atkinson, A., and Harrison, A. (1978). Distribution of personal wealth in Britain. Cambridge University Press, Cambridge.

[8] Bjerkedal, T. (1960). Acquisition of resistance in guinea pigs infected with different doses of virulent tubercle bacilli. American Journal of Epidemiol, 72(1), 130-148.

[9] Chahkandi, M., and Ganjali, M. (2009). On some lifetime distributions with decreasing failure rate, Computational Statistics and Data Analysis, 53(13), 4433-4440.

[10] Corbellini, A., Crosato, L., Ganugi, P. and Mazzoli, M. (2007). Fitting Pareto II distributions on firm size: Statistical methodology and economic puzzles. International Conference on Applied Stochastic Models and Data Analysis, Chania, Crete.

[11] Cordeiro, G. M., Ortega. E., and Popović, B. (2013). The gamma-Lomax distribution. Journal of Statistical Computation and Simulation, 85(2), 305-319.

[12] Ghitany, M. E., AL-Awadhi, F. A., and Alkhalfan, L. A. (2007) Marshall-Olkin extended Lomax distribution and its applications to censored data. Communication in Statistics Theory \& Methods, 36 (10), 1855-1866.

[13] Harris, C. M. (1968). The Pareto distribution as a queue service discipline. Operations Research, 16 (2), 307-313. 
[14] Hassan A. S. and Al-Ghamdi, A. (2009) Optimum step stress accelerated life testing for Lomax distribution. Journal of Applied Sciences Research, 5(12), 2153-2164.

[15] Hassan, A. S. and Abd-Alla, M. (2017). Exponentiated Lomax geometric distribution: Properties and applications. Paksitan Journal of Statistics \&Operation Resarch, 13(3), 545-566.

[16] Hassan, A. S., Assar, M. S. and Ali, K. A. (2016). The Compound Family of Generalized Inverse Weibull Power Series Distributions. British journal of Applied Sciences \& Technology, 14(3), 1-18.

[17] Hassan, A. S., Assar, M. S. and Shelbaia, A. (2016). Optimum step-stress accelerated life test plan for Lomax distribution with an adaptive type-II Progressive hybrid censoring. British journal of Mathematics \& Computer Science, 13(2),1-19.

[18] Holland, O., Golaup, A., and Aghvami, A. (2006). Traffic characteristics of aggregated module downloads for mobile terminal reconfiguration. In: IEE ProceedingsCommunications, 135(5), 683-690.

[19] Johnson, N. L., Kotz, S. and Balakrishnan, N. (1995). Continuous Univariate Distribution, Volume 2. 2nd edition. New York, Wiley and Sons.

[20] Kus, C., (2007). A new life time distribution. Computational Statistics and Data Analysis, 51(9), 4497-4509.

[21] Lemonte, A. J., and Cordeiro G.M. (2013). An extended Lomax distribution. Statistics, 47(4), 800-816.

[22] Lomax, K. S. (1954). Business failures: another example of the analysis of failure data. Journal of the American Statistical Association, 49, 847-852.

[23] Mahmoudi E., and Jafari, A. A. (2012). Generalized exponential-power series distributions. Computational Statistics and Data Analysis, 56(12),4047-4066.

[24] Morais, A. L., and Barreto-Souza, W. (2011). A compound family of Weibull and power series distributions. Computational Statistics and Data Analysis; 55(3), 1410-1425.

[25] Murthy, D.N.P. Xie, M. and Jiang, R. (2004). Weibull Models. Wiley.

[25] Rady, E. A., Hassanein, W. A. and Elhaddad, T. A. (2016). The power Lomax distribution with an application to bladder cancer data. SpringerPlus, https://www.ncbi.nlm.nih.gov/pubmed/27818876. 
[27] Ramos, M. W. A., Marinho, P. R. D., da Silva R. V. and Cordeiro, G. M. (2013). The exponentiated Lomax Poisson distribution with an application to lifetime data, Advances and Applications in Statistics, 34(2), 107-135.

[28] Roman, M., Louzada, F., Cancho, V. G., and Leite, J. G. (2012). A new long-term survival distribution for cancer data. Journal of Data Science, 10(2):241-258.

[29] Silva, R. B., Bourguignon, M., Dias, C. R. B., and Cordeiro, G. M. (2013). The compound family of extended Weibull power series distributions. Computational Statistics and Data Analysis, 58, 352-367.

[30] Tahir, M. H., Cordeiro, G. M., Mansoor, M. and Zubair, M. (2015). The Weibull-Lomax distribution: Properties and applications. Hacettepe Journal of Mathematics and Statistics, 44 (2), 461-480.

[31] Tahir, M. H., Hussain, M. A., Cordeiro, G. M., Hamedani, G. G., Mansoor, M. and Zubair, M. (2016). The Gumbel-Lomax distribution: Properties and applications. Journal of Statistical Theory and Applications, 15 (1), 61-79. 
Table 1: Results of simulation study of MSEs, RBs and SEs of estimates for different values of parameters $(\alpha, \lambda, \theta, \beta)$ for the power Lomax Poisson distribution

\begin{tabular}{|c|c|c|c|c|c|c|c|c|c|c|}
\hline \multirow[b]{2}{*}{$n$} & \multirow[b]{2}{*}{ Method } & \multirow[b]{2}{*}{ Properties } & \multicolumn{4}{|c|}{ Case I } & \multicolumn{4}{|c|}{ Case II } \\
\hline & & & $\alpha=0.75$ & $\begin{array}{l}\lambda \\
=0.5\end{array}$ & $\theta=1$ & $\beta=0.5$ & $\alpha=0.5$ & $\lambda=0.5$ & $\theta=1$ & $\beta=0.25$ \\
\hline \multirow{9}{*}{10} & \multirow{3}{*}{ ML } & MSE & 0.451 & 0.269 & 0.555 & 0.166 & 0.140 & 0.138 & 0.461 & 0.052 \\
\hline & & $\mathrm{RB}$ & 0.033 & 0.295 & 0.557 & 0.443 & 0.047 & 0.476 & 0.535 & 0.485 \\
\hline & & $\mathrm{SE}$ & 0.067 & 0.050 & 0.049 & 0.034 & 0.037 & 0.029 & 0.042 & 0.019 \\
\hline & \multirow{3}{*}{ LS } & MSE & 0.151 & 0.117 & 0.424 & 4.978 & 0.056 & 0.117 & 0.927 & 5.833 \\
\hline & & $\mathrm{RB}$ & 0.168 & 0.365 & 0.424 & 0.638 & 0.254 & 0.544 & 0.514 & 1.537 \\
\hline & & SE & 0.036 & 0.028 & 0.050 & 0.221 & 0.020 & 0.021 & 0.081 & 0.238 \\
\hline & \multirow{3}{*}{ WLS } & MSE & 0.162 & 0.110 & 0.455 & 4.744 & 0.057 & 0.106 & 0.681 & 5.849 \\
\hline & & $\mathrm{RB}$ & 0.206 & 0.393 & 0.416 & 0.619 & 0.231 & 0.531 & 0.516 & 1.603 \\
\hline & & SE & 0.038 & 0.028 & 0.052 & 0.215 & 0.021 & 0.019 & 0.064 & 0.239 \\
\hline \multirow{9}{*}{20} & \multirow{3}{*}{ ML } & MSE & 0.314 & 0.149 & 0.390 & 0.049 & 0.050 & 0.092 & 0.335 & 0.012 \\
\hline & & $\mathrm{RB}$ & 0.046 & 0.326 & 0.437 & 0.245 & 0.148 & 0.490 & 0.427 & 0.292 \\
\hline & & SE & 0.028 & 0.017 & 0.022 & 9.197* & 0.011 & $8.934^{*}$ & 0.020 & 4.064* \\
\hline & \multirow{3}{*}{ LS } & MSE & 0.084 & 0.087 & 0.245 & 0.040 & 0.102 & 0.097 & 0.324 & 0.083 \\
\hline & & $\mathrm{RB}$ & 0.180 & 0.362 & 0.335 & 0.175 & 0.186 & 0.466 & 0.428 & 0.308 \\
\hline & & SE & 0.013 & 0.012 & 0.018 & $8.932 *$ & 0.015 & 0.010 & 0.019 & 0.014 \\
\hline & \multirow{3}{*}{ WLS } & MSE & 0.126 & 0.089 & 0.295 & 0.039 & 0.065 & 0.092 & 0.346 & 0.082 \\
\hline & & $\mathrm{RB}$ & 0.104 & 0.299 & 0.359 & 0.160 & 0.143 & 0.434 & 0.442 & 0.277 \\
\hline & & SE & 0.018 & 0.013 & 0.020 & $9.105 *$ & 0.012 & 0.011 & 0.019 & 0.014 \\
\hline \multirow{9}{*}{30} & \multirow{3}{*}{ ML } & MSE & 0.118 & 0.108 & 0.297 & 0.028 & 0.030 & 0.082 & 0.281 & $8.673^{*}$ \\
\hline & & RB & 0.134 & 0.386 & 0.371 & 0.204 & 0.171 & 0.482 & 0.385 & 0.244 \\
\hline & & $\mathrm{SE}$ & 0.011 & $8.890 *$ & 0.013 & $4.363 *$ & $5.070 *$ & $5.118^{*}$ & 0.012 & $2.343^{*}$ \\
\hline & \multirow{3}{*}{ LS } & MSE & 0.083 & 0.078 & 0.229 & 0.026 & 0.025 & 0.070 & 0.256 & 0.016 \\
\hline & & RB & 0.184 & 0.366 & 0.318 & 0.162 & 0.195 & 0.429 & 0.349 & 0.216 \\
\hline & & SE & $8.399 *$ & $7.059 *$ & 0.012 & $4.707 *$ & $4.213 *$ & $5.160 *$ & 0.012 & $3.740 *$ \\
\hline & \multirow{3}{*}{ WLS } & MSE & 0.081 & 0.075 & 0.248 & 0.024 & 0.029 & 0.070 & 0.290 & $7.253 *$ \\
\hline & & $\mathrm{RB}$ & 0.131 & 0.315 & 0.330 & 0.143 & 0.141 & 0.393 & 0.398 & 0.182 \\
\hline & & SE & 9.024* & $7.507 *$ & 0.012 & $4.574 *$ & $5.209 *$ & $5.915^{*}$ & 0.012 & 2.417 * \\
\hline \multirow{9}{*}{50} & \multirow{3}{*}{ ML } & MSE & 0.056 & 0.063 & 0.208 & 0.016 & 0.027 & 0.087 & 0.243 & $5.470 *$ \\
\hline & & $\mathrm{RB}$ & 0.145 & 0.380 & 0.280 & 0.155 & 0.177 & 0.471 & 0.337 & 0.213 \\
\hline & & SE & $4.206^{*}$ & $3.281 *$ & $7.195 *$ & $2.002 *$ & $2.779 *$ & $3.555^{*}$ & $7.190 *$ & $1.025^{*}$ \\
\hline & \multirow{3}{*}{ LS } & MSE & 0.070 & 0.064 & 0.173 & 0.017 & 0.021 & 0.069 & 0.230 & 5.777 * \\
\hline & & $\mathrm{RB}$ & 0.156 & 0.322 & 0.254 & 0.127 & 0.193 & 0.436 & 0.343 & 0.195 \\
\hline & & $\mathrm{SE}$ & $4.745^{*}$ & $3.896^{*}$ & $6.597 *$ & $2.238 *$ & $2.135 *$ & $2.940^{*}$ & $6.709 *$ & $1.165^{*}$ \\
\hline & & MSE & 0.057 & 0.058 & 0.191 & 0.015 & 0.022 & 0.067 & 0.280 & $5.612 *$ \\
\hline & WLS & $\mathrm{RB}$ & 0.123 & 0.299 & 0.268 & 0.116 & 0.147 & 0.408 & 0.392 & 0.182 \\
\hline & & SE & $4.449 *$ & $3.774 *$ & $6.893 *$ & $2.128 *$ & $2.623 *$ & $3.183^{*}$ & $7.179 *$ & $1.192 *$ \\
\hline & & MSE & 0.031 & 0.044 & 0.134 & $8.447 *$ & 0.018 & 0.065 & 0.168 & $2.932 *$ \\
\hline & ML & $\mathrm{RB}$ & 0.160 & 0.354 & 0.187 & 0.117 & 0.193 & 0.460 & 0.249 & 0.165 \\
\hline & & SE & $1.295^{*}$ & $1.126^{*}$ & $3.139 *$ & $0.710 *$ & $0.912 *$ & $1.109 *$ & $3.260 *$ & $0.350^{*}$ \\
\hline & & MSE & 0.034 & 0.042 & 0.105 & 0.010 & 0.016 & 0.057 & 0.178 & $3.744 *$ \\
\hline 100 & LS & $\mathrm{RB}$ & 0.173 & 0.315 & 0.176 & 0.106 & 0.181 & 0.403 & 0.291 & 0.160 \\
\hline & & $\mathrm{SE}$ & $1.298^{*}$ & $1.318 *$ & $2.727 *$ & $0.854 *$ & $0.909 *$ & $1.284 *$ & $3.051 *$ & $0.463^{*}$ \\
\hline & & MSE & 0.033 & 0.040 & 0.133 & $8.847 *$ & 0.016 & 0.056 & 0.212 & $3.118^{*}$ \\
\hline & WLS & $\mathrm{RB}$ & 0.138 & 0.292 & 0.204 & 0.095 & 0.153 & 0.394 & 0.329 & 0.148 \\
\hline & & SE & $1.512 *$ & $1.367 *$ & $3.038 *$ & $0.813 *$ & $0.996^{*}$ & $1.314^{*}$ & $3.241 *$ & $0.420 *$ \\
\hline
\end{tabular}

\footnotetext{
* Indicate that the value multiply $10^{-3}$
} 
Table 2: Results of simulation study of MSEs, RBs and SEs of estimates for different values of parameters $(\alpha, \lambda, \theta, \beta)$ for the power Lomax Poisson distribution

\begin{tabular}{|c|c|c|c|c|c|c|}
\hline \multirow{2}{*}{$n$} & \multirow{2}{*}{ Method } & \multirow{2}{*}{ Properties } & \multicolumn{4}{|c|}{ Case III } \\
\hline & & & $\alpha=0.75$ & $\lambda=0.5$ & $\theta=1$ & $\beta=0.5$ \\
\hline \multirow{9}{*}{10} & \multirow{3}{*}{ ML } & MSE & 0.266 & 0.617 & 0.894 & 0.736 \\
\hline & & $\mathrm{RB}$ & 0.252 & 0.117 & 0.921 & 1.175 \\
\hline & & SE & 0.051 & 0.078 & 0.022 & 0.082 \\
\hline & \multirow{3}{*}{$\mathrm{LS}$} & MSE & 0.123 & 0.229 & 0.753 & 0.734 \\
\hline & & $\mathrm{RB}$ & 0.025 & 0.388 & 0.838 & 0.829 \\
\hline & & SE & 0.035 & 0.044 & 0.022 & 0.084 \\
\hline & \multirow{3}{*}{ WLS } & MSE & 0.130 & 0.245 & 0.790 & 2.398 \\
\hline & & RB & 0.063 & 0.337 & 0.847 & 1.181 \\
\hline & & SE & 0.036 & 0.047 & 0.027 & 0.153 \\
\hline \multirow{9}{*}{20} & \multirow{3}{*}{ ML } & MSE & 0.056 & 0.236 & 0.800 & 0.037 \\
\hline & & RB & $7.756^{*}$ & 0.352 & 0.887 & 0.484 \\
\hline & & SE & 0.012 & 0.023 & $5.729 *$ & $8.267 *$ \\
\hline & \multirow{3}{*}{$\mathrm{LS}$} & MSE & 0.034 & 0.125 & 0.690 & 0.043 \\
\hline & & $\mathrm{RB}$ & 0.050 & 0.438 & 0.821 & 0.375 \\
\hline & & SE & $9.219 *$ & 0.014 & $6.261 *$ & $9.644 *$ \\
\hline & \multirow{3}{*}{ WLS } & MSE & 0.049 & 0.149 & 0.704 & 0.035 \\
\hline & & RB & 0.016 & 0.365 & 0.830 & 0.332 \\
\hline & & SE & 0.011 & 0.017 & $6.087 *$ & $8.809 *$ \\
\hline \multirow{9}{*}{30} & \multirow{3}{*}{ ML } & MSE & 0.038 & 0.171 & 0.802 & 0.011 \\
\hline & & $\mathrm{RB}$ & $7.566^{*}$ & 0.407 & 0.890 & 0.300 \\
\hline & & SE & $6.527 *$ & 0.012 & $3.334 *$ & $2.933^{*}$ \\
\hline & \multirow{3}{*}{ LS } & MSE & 0.018 & 0.098 & 0.675 & $9.967 *$ \\
\hline & & $\mathrm{RB}$ & 0.056 & 0.455 & 0.814 & 0.262 \\
\hline & & SE & 4.403* & $7.140 *$ & $3.759 *$ & $2.833^{*}$ \\
\hline & \multirow{3}{*}{ WLS } & MSE & 0.030 & 0.126 & 0.693 & $8.666^{*}$ \\
\hline & & RB & 0.014 & 0.377 & 0.824 & 0.233 \\
\hline & & SE & $5.820 *$ & 0.010 & $3.977 *$ & $2.695^{*}$ \\
\hline \multirow{9}{*}{50} & \multirow{3}{*}{ ML } & MSE & 0.012 & 0.097 & 0.782 & $5.261 *$ \\
\hline & & RB & 0.050 & 0.499 & 0.879 & 0.233 \\
\hline & & SE & $2.116^{*}$ & $3.730 *$ & $1.927 *$ & $1.111^{*}$ \\
\hline & \multirow{3}{*}{$\mathrm{LS}$} & MSE & 0.014 & 0.085 & 0.643 & $6.057 *$ \\
\hline & & RB & 0.046 & 0.452 & 0.794 & 0.223 \\
\hline & & SE & $2.310^{*}$ & $3.704 *$ & $2.184 *$ & $1.275^{*}$ \\
\hline & \multirow{3}{*}{ WLS } & MSE & 0.014 & 0.079 & 0.667 & $5.197 *$ \\
\hline & & RB & $5.024 *$ & 0.404 & 0.809 & 0.183 \\
\hline & & SE & $2.409^{*}$ & $3.959 *$ & $2.292 *$ & $1.252 *$ \\
\hline \multirow{9}{*}{100} & \multirow{3}{*}{ ML } & MSE & $7.551^{*}$ & 0.090 & 0.781 & $2.859^{*}$ \\
\hline & & RB & 0.059 & 0.517 & 0.878 & 0.187 \\
\hline & & SE & $0.844^{*}$ & $1.512 *$ & $0.966 *$ & $0.382 *$ \\
\hline & & MSE & $6.474^{*}$ & 0.066 & 0.607 & $3.437 *$ \\
\hline & LS & $\mathrm{RB}$ & 0.037 & 0.436 & 0.772 & 0.172 \\
\hline & & SE & 0.794* & $1.348 *$ & $1.021 *$ & $0.475^{*}$ \\
\hline & & MSE & $8.821^{*}$ & 0.064 & 0.631 & $2.773^{*}$ \\
\hline & WLS & $\mathrm{RB}$ & $4.887 *$ & 0.398 & 0.787 & 0.143 \\
\hline & & SE & $0.950 *$ & $1.583^{*}$ & $1.089 *$ & $0.446^{*}$ \\
\hline
\end{tabular}

* Indicate that the value multiply $10^{-3}$ 
${ }^{1}$ Amal S. Hassan

Department of Mathematical Statistics

Institute of Statistical Studies \& Research

Cairo University, Giza city, 12613, Egypt

E-mail ID: dr.amalelmoslamy@gmail.com

${ }^{2}$ Said G. Nassr

Department of Quantitative Methods

Faculty of Business Administration and International Marketing

Sinai University, El-Arish city, North Sinai, 16020, Egypt

E-mail ID: dr.saidstat@gmail.com 
